\title{
TWO DIMENSIONAL WAKE VORTEX SIMULATIONS IN THE ATMOSPHERE: PRELIMINARY SENSITIVITY STUDIES
}

\author{
F.H. Proctor ${ }^{\star}$ and D.A. Hinton ${ }^{\dagger}$ \\ NASA Langley Research Center \\ Flight Dynamics \& Control Division \\ Hampton, VA 23681-0001 \\ J. Han ${ }^{*}$ D.G. Schowalter ${ }^{\S}$, and Y.-L. Lin ${ }^{\mathbb{I I}}$ \\ Department of Marine, Earth \& Atmospheric Science \\ North Carolina State University \\ Raleigh, NC 27695-8208
}

\begin{abstract}
A numerical large-eddy simulation model is currently being used to quantify aircraft wake vortex behavior with meteorological observables. The model, having a meteorological framework, permits the interaction of wake vortices with environments characterized by crosswind shear, stratification, and humidity. The addition of grid-scale turbulence as an initial condition appeared to have little consequence. Results show that conventional nondimensionalizations work very well for vortex pairs embedded in stably stratified flows. However, this result is based on simple environments with constant Brunt-Vaisala frequency. Results presented here also show that crosswind profiles exert important and complex interactions on the trajectories of wake vortices. Nonlinear crosswind profiles tended to arrest the descent of wake vortex pairs. The member of the vortex pair with vorticity of same sign as the vertical change in the ambient along-track vorticity may be deflected upwards.
\end{abstract}

\section{Introduction}

As an element of NASA's Terminal Area Productivity (TAP) program, a significant effort is underway at NASA Langley to develop a system that will provide dynamical aircraft vortex spacing criteria to Air Traffic Control (ATC) automation. The system, called the Aircraft Vortex Spacing System ${ }^{1,2}$ (AVOSS), will determine safe operating spacings between arriving/departing aircraft based on the observed/predicted weather state. In order to develop this system, research is being focused toward understanding how wake vortices interact with the atmosphere. The objectives of this research are to quantify wake vortex

${ }^{*}$ Research Scientist, AIAA member

${ }^{\dagger}$ AVOSS Team Group Leader

${ }^{\dagger}$ Research Assistant

${ }^{\S}$ Visiting Assistant Professor

"Associate Professor behavior as related to the atmospheric conditions using validated numerical simulations, as well as observed data from recent and on-going field studies. NASA-Langley theoretical modelling efforts are expected to play a major role in the development of these algorithms.

Presented in this paper are results from a numerical large-eddy simulation model called the Terminal Area Simulation System ${ }^{3,4}$ (TASS) which has been adapted for application to aircraft wake vortex simulations. The TASS model has a meteorological reference frame, a compressible non-Boussinesq equation set, subgrid turbulence closure, and a formulation for ground-friction. The TASS model is capable of simulating post roll-up wake vortices in both two and three dimensions. The model is applicable to a wide range of atmospheric conditions that include: vertical wind shear, stratification, atmospheric boundary layer turbulence, fog, and precipitation. Ongoing research using the two-dimensional (2-D) version of this model is presented in this paper while results using the

Copyright (C) 1997 by the American Institute of Aeronautics, Inc. No copyright is asserted in the United States under Title 17, U.S. Code. The U.S. Government has a royalty-free license to exercise all rights under the copyright claimed herin for government purposes. All other rights are reserved by the copyright owner. 
three-dimensional (3-D) version are presented in an accompanying paper. ${ }^{5}$ In a recent study we were able to achieve excellent comparisons of simulated vortex trajectories with field data. ${ }^{4}$ We are now using 2-D TASS to investigate how meteorology affects vortex transport -knowledge of which is crucial to the development and implementation of the AVOSS system. The advantage of the 2-D system is its ease in use and the relatively small amount of computer resources required per run. Our early investigation is primarily focused on understanding how stratification, crosswinds, and ground proximity affect the trajectory of wake vortices. We intend to use 3-D TASS to investigate vortex decay, vortex interaction with atmospheric turbulence, and the onset of three dimensional instabilities.

The TASS model results will be applied to the specific requirements of the AVOSS system design. In particular, prediction of the motion of wake vortices from initial generation until departure from the approach corridor is critical, while motion beyond the approach corridor is less critical. The dimensions of the AVOSS approach corridor are described in Hinton. ${ }^{1,2}$ Laterally the corridor is 91.4 meters wide along the runway and out to a distance of about 859 meters from the runway. This is the location of the approach middle marker transmitter. The corridor lateral limits then widen to a width of 305 meters at a distance of 9272 meters $(5 \mathrm{~nm})$ from the runway. This is a typical location for the approach outer marker, where approaching aircraft transition from level flight to the glide slope. The approach corridor vertical dimension places a floor at ground level along the runway out to the middle marker location. Currently two options exist for the AVOSS corridor floor, and may be altered based on industry input. The most conservative option provides a floor that begins at ground level at the middle marker and rises at a shallower angle than the glide slope, so that the floor is 122 meters (400 feet) below the glide slope at the outer marker. No reduced separation credit is given for vertical wake motion very near the runway, so detailed wake bounce studies at this location are not required at this time. There is no vertical corridor limit above the glide slope, since vertical rising of the wakes is not assumed to be a viable mechanism for reduced separation of aircraft.

The requirements for AVOSS simplifies our task, somewhat, by limiting the application of our experiments to a confined region defined by the flight corridor. Any vortex that has drifted outside these lateral or vertical limits is no longer considered a factor to a following aircraft. The relationships determined with the TASS model must lead to simple rules or analytical models that can reliably and accurately predict the time required for the wakes from many aircraft types to leave the corridor, in a wide range of atmospheric conditions. In weather situations that cannot reliably remove the wakes from the corridor in a period of about 1 to 2 minutes, the AVOSS system will either rely on decay predictions to reduce separations or maintain separation at the current standards in use today. Provided there is assurance that a wake cannot move back into the corridor, neither, the AVOSS system, nor the numerical models need to predict wake motion outside of the corridor. In determining wake sensitivity to various parameters this small range of motions must be considered. Application of AVOSS to parallel runway operations, however, may require study at a later date of wake drift over lateral distances of 400 to 900 meters as well as more detailed vertical motion studies at very low altitude.

\section{Previous Research}

Many investigations, including experimental, theoretical, and field studies, of wake vortex phenomenon have been conducted over the past three decades. Important processes that affect wake vortex transport are well known and include stratification, turbulence, ground interaction, and ambient crosswind.

Numerous theoretical and experimental studies have examined the effect of stratification on wake vortices. $6,7,8,9,10,11,12,13,14,15$ The primary processes of how stable stratification affects the descent of wake vortices is well known. As a wake vortex pair descends due to the mutual induction of its circulation fields, temperature and buoyancy forces increase due to adiabatic compression. The positive buoyancy forces act to diminish the descent rate with time. The descending volume of fluid that encompasses the wake vortex pair, termed the vortex oval, develops temperature differences along its interface which act to generate counter-sign vorticity. Unresolved issues remain, such as whether the spacing between the vortices increases or decreases with time, and whether there is there a limiting descent altitude based on the magnitude of stratification.

Another characteristic of the atmosphere which can affect the motion of wake vortices is vertical shear of the ambient winds. Although wind shear is commonly known to occur near the ground due to the effects of surface drag, it exists at other altitudes due to vertical changes in horizontal gradients of atmospheric pressure. In addition, significant vertical changes of the wind can be found along fronts and inversions. For example, 
strong vertical shears can exist along typical nocturnal inversions that divide stable air (produced by radiative cooling at the ground) and the residual atmospheric boundary layer air that lies above. ${ }^{16}$ Vertical change in the crosswind, or "crosswind shear" is known to have some peculiar effects on wake vortices. From field measurements Brashears et al. ${ }^{17}$ reported that crosswind shear acted to reduce the vortex sink rates. The reduction in sink rate was usually greater for the downstream vortex, resulting in tilting of the vortex pair. However, for strong shears there was an opposite trend, with the upstream vortex sometimes seen to rise. Most numerical investigators have concentrated on studying the effects of crosswind shear when the wake vortices were generated near the ground. ${ }^{10,18,19}$ Generally they see a tilting (rotation) of the vortex pair in a direction opposite to that of the shear vorticity, with the downwind (downshear) vortex being at greater altitude than the upwind (upshear) vortex. Laboratory experiments by Delisi et al. ${ }^{20}$ and numerical experiments by Robins and Delisi ${ }^{14}$ show that strongly-stratified shear flow can tilt the wake vortex pair and lead to a solitary vortex. The vortex having vorticity opposite to that of the shear vorticity was dissipated leaving a strong solitary vortex with rotation in the same sense as the ambient shear. Robins and Delisi believed that the accelerated weakening of one of the vortices was responsible for the tilting of the vortex pair. Recent numerical experiments by Schilling ${ }^{21}$ and Proctor ${ }^{4}$ suggest that the influence of stratification on the vortex trajectories is secondary to crosswind shear, at least for wakes generated below 175 meters.

\section{Numerical Model}

The TASS model is a multi-dimensional, largeeddy code developed within a meteorological framework. Grid-scale turbulence is explicitly computed while the effects of subgrid-scale turbulence are modeled by a Smagorinsky closure model that is modified for stratification. The TASS model has parameterizations for ground stresses that are a function of surface roughness, allowing for "in-ground effect" wake simulations with realistic ground interactions. The TASS model consists of 12 prognostic equations: three equations for momentum, one equation each for pressure deviation and potential temperature, six coupled equations for continuity of water substance (water vapor, cloud droplet water, cloud ice crystals, rain, snow and hail) and a prognostic equation for a massless tracer. The lateral boundary condition may be open or periodic, with the open condition utilizing a mass-conservative, nonreflective, radiation boundary scheme. Explicit numerical schemes are used in TASS that are quadratic conservative, and have almost no numerical diffusion. ${ }^{22}$ Ambient atmospheric conditions are initialized with a vertical profile of temperature, dew point, and wind velocity. The two dimensional simulations are initialized with a simple vortex system representative of the post roll-up velocity field. Details of the TASS model and wake initialization can be found in Proctor. ${ }^{4}$

All of the cases except those in Section VI assume that there is no preexisting grid-scale turbulence. As mentioned previously, subgrid-scale turbulence is treated by first-order closure. Section VI examines the sensitivity to grid-scale turbulence.

\section{Stratification}

The purpose of this set of experiments is to see how well TASS 2-D simulations compare with laboratory data for the case of wake vortices in a very stably stratified environment. The second goal of this set experiments it see how normalized results from different aircraft intercompare. The experiments are run with dimensional variables with the results normalized for space and time scales following Sarpkaya ${ }^{12}$ as:

$$
Z^{*}=\frac{z}{b_{o}}, \quad T^{*}=t \frac{\Gamma_{o}}{2 \pi b_{o}^{2}}
$$

where $Z^{*}$ is the nondimensional height, $z$ dimensional height, $b_{o}$ initial vortex spacing, $T^{*}$ nondimensional time, $t$ dimensional time, and $\Gamma_{o}$ the initial circulation. The ambient lapse rate for temperature can be specified in terms of the nondimensional Brunt-Vaisala frequency as:

$$
N^{*}=\frac{N}{T^{*}}=\frac{2 \pi N b_{o}^{2}}{\Gamma_{o}}
$$

where the dimensional Brunt-Vaisala frequency $N$, is related to temperature $\tau$, and potential temperature $\theta$ (which is conserved for dry adiabatic processes):

$$
N^{2}=\frac{g}{\theta} \frac{\partial \theta}{\partial z} \simeq \frac{g}{\tau}\left[\frac{\partial \tau}{\partial z}+\frac{g}{c_{p}}\right]
$$

where $g$ is the acceleration due to gravity, and $c_{p}$ is the specific heat of air at constant pressure. Note, that if we assume the vortex separation remains constant, inviscid theory predicts that a vortex pair will descend to a maximum depth of order: $Z^{*}=\mathcal{Q}\left(1 / N^{*}\right)$; and for neutral stratification the vortex pair descends $Z^{*}=1$ in time $T^{*}=1$. 
Table 1. Aircraft and parameters for $N^{*}=1$ comparisons.

\begin{tabular}{cccc}
\hline Aircraft & $\boldsymbol{b}_{\boldsymbol{o}}(\mathbf{m})$ & $\Gamma_{\boldsymbol{o}}\left(\mathbf{m}^{2} / \mathbf{s}\right)$ & $\boldsymbol{N}\left(\mathbf{1 0}^{-3} \mathbf{s}^{-1}\right)$ \\
\hline Fokker 28 & 19.69 & 160.8 & 66 \\
\hline B 737-200 & 22.26 & 205.5 & 66 \\
\hline B 757-200 & 29.80 & 306.9 & 55 \\
\hline DC 10-10 & 37.18 & 573.0 & 66 \\
\hline DC 10-30 & 39.60 & 492.7 & 50 \\
\hline EA 330 & 46.78 & 378.1 & 27.5 \\
\hline B 747-400 & 50.51 & 534.3 & 33.3 \\
\hline
\end{tabular}

\section{$\underline{\text { Initial Conditions }}$}

The list of aircraft used in this set of experiments is shown in Table 1. The initial circulations $\left(\Gamma_{o}\right)$ are based on typical weights and approach speeds for each specific aircraft. [For the $D C-10-10$ we assumed a higher weight than average, but within the maximum landing weight.] Each case assumes a lapse rate of temperature such that $N^{*}=1$. Although $N^{*}$ is fixed, the values of $N$ differ for each airplane due to different initial vortex spacings $\left(b_{o}\right)$ and circulations (e.g., see Eq. 1). The lapse rate for potential temperature needed to achieve $N^{*}=1$ ranges from about 2.3 times greater than for isothermal conditions for the Airbus 330 to over 13 times greater than isothermal for the Fokker 28-4000, Boeing 737-200, and $D C$ 10-10. Although the temperature lapse rates for some of the cases are unrealistically large, the set of experiments is useful in examining how nondimensionalized results compare for vortices generated by different types of aircraft.

The experiments also assume a compressible atmosphere in which density and pressure decrease with height in accordance with the specified lapse rate for temperature. The domain width and depth is $4 b_{o}$ and $6 b_{o}$, respectively. The vortex generation height is approximately $3.5 b_{o}$ above the ground. A constant grid resolution of $b_{o} / 50.5$ is assumed in both vertical and horizontal directions. The model environments contain neither ambient wind nor grid-scale turbulence.

\section{$\underline{\text { Results and Comparisons }}$}

Results of the seven experiments are shown in Fig. 1. The non-dimensionalized descent rates for all seven are nearly identical and agree well Sarpkaya's laboratory measurements conducted in water at moderate

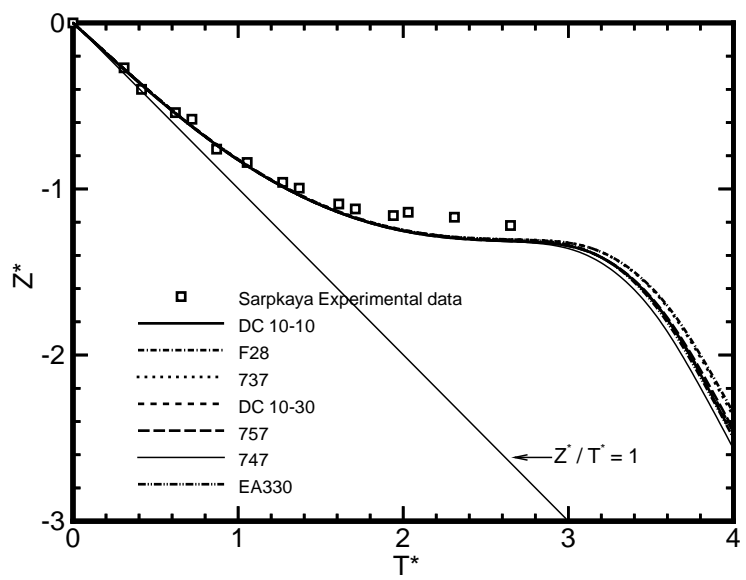

Figure 1. Aircraft sensitivity to nondimensional height and time for $N^{*}=1$. TASS simulations for 7 aircraft (lines) compared with Sarpkaya's experimental data ${ }^{12}$ (squares). Curve for $d Z^{*} / d T^{*}=1$ (thin line) shown also.

Reynold's number for a delta-wing aircraft. ${ }^{12}$ The results indicate that the vortex pair initially descend at $d Z^{*} / d T^{*}=1$, as is true in Sarpkaya's data. However, as the buoyancy forces increase due to compressional heating the descent rate slows, until at $T^{*}=2$, the vortex descent is halted. The lateral separation distance (not shown) between the two counter-rotating vortices remained nearly constant in the TASS simulations, then began to close together after $T^{*}=2.5$. This reduction in separation forced the simulated vortices to descend once again. Sarpkaya's experiments, which were at moderate Reynold's numbers, were unable track the weakening vortex pair after $T^{*}=3$. Spalart ${ }^{15}$ recently published results from 2-D laminar simulations, which showed his vortices pausing then ascending after $T^{*}=2.25$, before again descending after $T^{*}=4$. TASS simulation results presented by Schowalter et al. ${ }^{5}$ suggest that threedimensional effects begin to influence the vortex after $T^{*}=3$, and the vortex descent following that time might be an artifact of the 2-D assumption.

Our model results for $N^{*}=1$ show that the stable stratification influences the descent of the vortex pair in 
following way. Air from near the generation height is transported downward within the vortex oval (Fig. 2) and heats due to adiabatic compression. The increase in positive buoyancy from compressional heating has the most significant impact on the weaker tangential velocities at larger radii. Eventually the tangential velocities (and circulation) at radii greater than $1 / 2 b_{o}$ are diminished. This weakens the mutual interaction of the vortices, and eventually causes them to stall (at $\sim 1.25 b_{o}$ below the generation height for $N^{*}=1$ ). Furthermore, our 2-D simulations at $N^{*}=1$, show that eddies generated

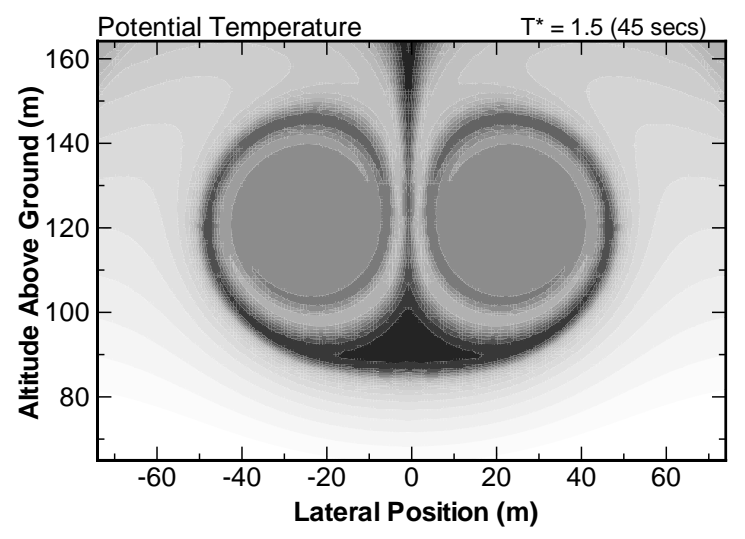

Figure 2. Vertical cross section of the potential temperature filed associated with a descending vortex pair in stratified fluid. Darker colors represent warmer temperatures and delineate the vortex oval from the surrounding fluid.

baroclinically at the vortex-oval periphery are transported upward and pinch the vortex pair closer together, resulting in the downward acceleration seen after $T^{*}=3$.

Another observation to be made from this set of experiments is the sensitivity of aircraft type to lapse rate. Although we have yet to do an extensive sensitivity study on the effects of stratification, it is obvious that wake vortices produced by large, wide-body aircraft such as the $B-747$ and $E A-330$ are much more sensitive to stable stratification than those produced from medium and light commercial jetliners. Mostly due to their smaller wingspans, rather large lapse rates are needed for the small and medium sized aircraft to achieve the same value for $N^{*}$ as that for the "Jumbos."

\section{Crosswind Shear}

Although a number of researchers have examined the effects of crosswind shear on wake vortices, they usually have confined their experiments either to regions either close to the ground or simple shear profiles. In the experiments presented here, we look at the effect of narrow shear zones, such as one may find

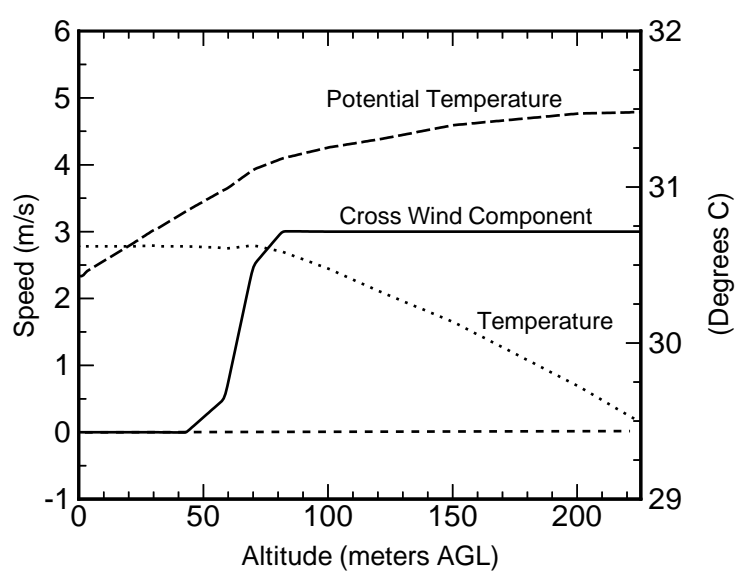

Figure 3. Initial profiles for ambient temperature, potential temperature, and crosswind, for the shearlayer sensitivity experiments. [Only the wind profile for the $3 \mathrm{~m} / \mathrm{s}$ crosswind change is show.]

near a thermal inversion. In a second set of experiments, we examine wake vortex sensitivity to several types of shear profiles. For both sets of experiments we assume dimensional variables with initial wake conditions based on a B-727-100 aircraft (Table 2).

\section{$\underline{\text { Sensitivity to Shear Layers. }}$}

The motivation behind the following set of experiments was our observation of Memphis 1994 and 1995 field data. Following sunset, many wake vortices tended either to stall or to be deflected when penetrating a region near the top of a low-level temperature inversion. ${ }^{23}$ This inversion separated the radiativelycooled air near the ground and the near-neutral residual boundary layer above. The measured levels of stratification, alone, were too weak to cause the observed levels of deflection. We postulate that pronounced shear can exist in the inversion zone due to changes in airmass characteristics. Unfortunately, high resolution wind data was not always available near the altitude of the inversion to confirm our suspicions.

In order to test the effect of shear zones on wake vortex descent, several experiments were conducted as 
follows. An isothermal temperature profile was assumed below an altitude of $75 \mathrm{~m}$, above which it transitions to a slightly-stable profile. Wind profiles were assumed to have no crosswind below an altitude of $50 \mathrm{~m}$ transitioning to a constant crosswind value above $80 \mathrm{~m}$ (e.g., Fig. 3). Hence, the crosswind change over $30 \mathrm{~m}$ was also equal to the crosswind above $80 \mathrm{~m}$.

Six experiments were run with the initial conditions listed in table 2 , with crosswind changes of: 0 , $1,2,3,4$ and $8 \mathrm{~m} / \mathrm{s}$. In order to evaluate any effects that the stable temperature stratification may be having, an additional experiment is conducted assuming a neutral temperature profile. An example of the profile for the $3 \mathrm{~m} / \mathrm{s}$ crosswind change can be seen in Fig. 3 .

A comparison of altitude vs lateral position for this set of experiments shows the sensitivity of the vortex trajectories to the shear zone (Fig.4). The descending wake vortices that encountered crosswind changes greater than $1 \mathrm{~m} / \mathrm{s}$ were deflected downstream. Stronger shears also resulted in larger separations between the upstream

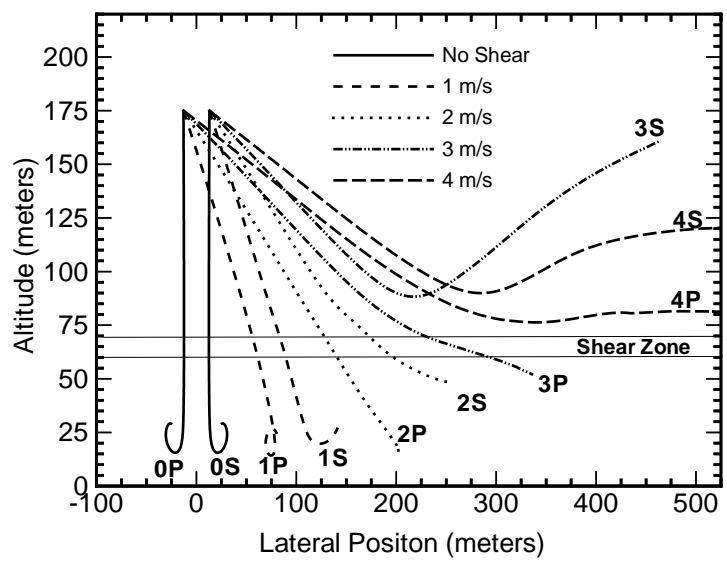

Figure 4. Trajectories for the simulated wake vortices from the shear zone sensitivity experiments. Both port $(P)$ and starboard $(S)$ trajectories are shown for each shear profile. [The Starboard vortex $(S)$ is downstream from the port vortex.]

and downstream vortex. Crosswind changes of $3 \mathrm{~m} / \mathrm{s}$ and larger were sufficiently strong to block the downward penetration of the vortices. The time height trajectories in Figs 5 and 6 show that a $2 \mathrm{~m} / \mathrm{s}$ or greater crosswind shear significantly suppresses the sink rate of the vortices. Also, for a $3 \mathrm{~m} / \mathrm{s}$ or greater shear the downstream vortex is deflected upwards.
Table 2. Assumed values for initial parameters.

\section{Initial Conditions}

\begin{tabular}{lc}
\hline Parameter & Value \\
\hline Generation Height & $175 \mathrm{~m}$ \\
Circulation $\left(\Gamma_{\mathrm{o}}\right)$ & $250 \mathrm{~m}^{2} \mathrm{~s}^{-1}$ \\
Vortex Spacing $\left(\mathrm{b}_{0}\right)$ & $26 \mathrm{~m}$ \\
Core Radius & $1.75 \mathrm{~m}$ \\
Numerical Grid Size & $0.75 \mathrm{~m}$ \\
\hline
\end{tabular}

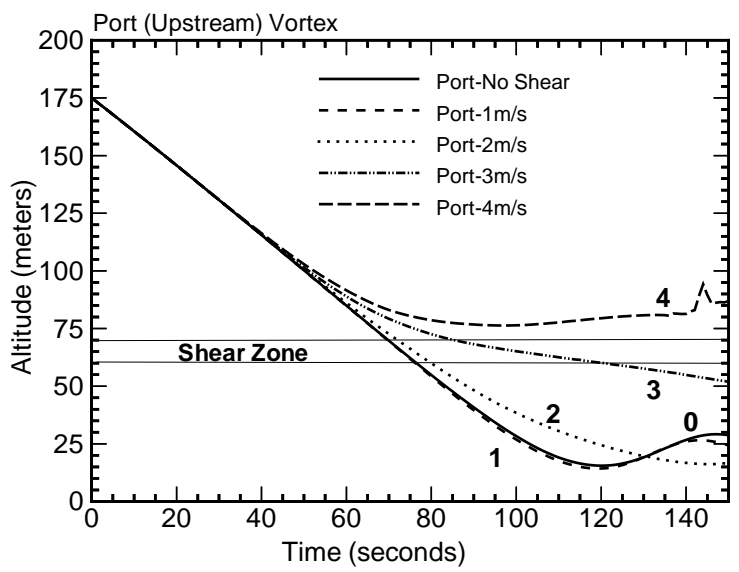

Figure 5. Altitude vs time trajectory for upstream vortices in shear zone sensitivity experiments.

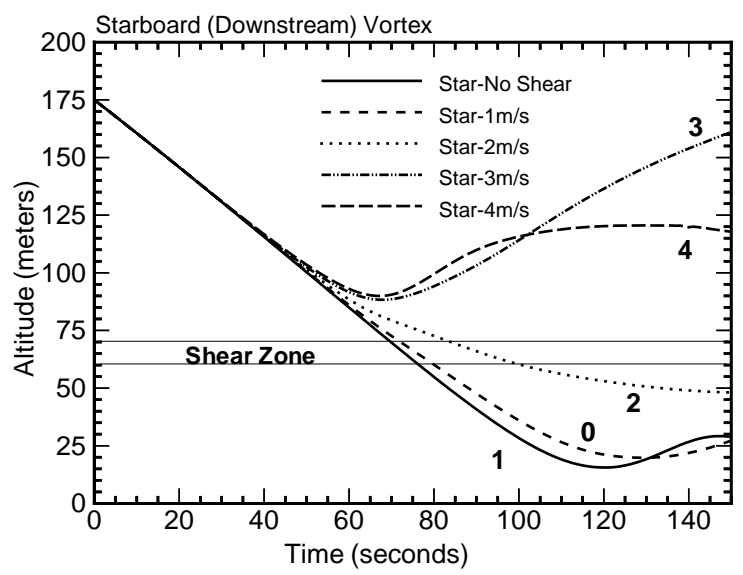

Figure 6. Same as Fig. 5, but downstream vortices. 
The assumed crosswind shear profiles had the most direct influence on the downstream (starboard) vortex. As indicated in Figs. 4-6, this vortex was more sensitive and deflected to a higher altitude than its upstream counterpart. The deflection of the downstream vortex increased the separation distance from the upstream (port) vortex, thus weakening the mutual induction between the two vortices (see Fig. 4). Therefore, for the stronger shear cases the upstream vortex was left to drift near the altitude of the shear zone.

The mechanism responsible for this deflection is unclear to us at this time. The downstream vortex contains vorticity of opposite sign to that of the shear. However, there was no detectable preference for the downstream (or upstream) vortex to weaken at a greater rate.

An additional experiment was run with a neutral atmosphere rather than the temperature profile in Fig. 3. It gave nearly identical results to the experiment which assumed the sounding in Fig. 3, which implies that stratification played no role in the modification of the vortex trajectories. Note from Eq. (1) and assuming the specifications in Table 2, the ambient temperature would have to increase with altitude at $3.25^{\circ} \mathrm{C}$ per 35 meters in order to suppress vortex descent within a $35 \mathrm{~m}$ thick zone.

A last point remains to be made about the trajectories in Fig. 4 for the $1 \mathrm{~m} / \mathrm{s}$ and $2 \mathrm{~m} / \mathrm{s}$ shear cases. There is a continued cross track drift of the vortices after they have descended below the shear layer into the air mass with no ambient crossflow. This effect is due to the downward transport of crossflow momentum with the descending vortex oval. Like potential temperature as shown in Fig. 2, crossflow momentum is transported from altitudes near the generation height (i.e. flight path). The consequence of this finding is that wind measurements near the surface may not always correlate with the lateral transport of the wake vortices.

\section{$\underline{\text { Additional Crosswind Shear Experiments }}$}

To provide further insight into the role that shear may have on vortex transport, a second set of experiments are presented. This set consists of four experiments that are designed to test wake vortex sensitivity to environments with linear shear and transitioning shear. As with the previous set, the experiments assume the initial parameters in Table 2, but with neutral stratification and with the wind profiles shown in Fig. 7. Included, is a baseline experiment with no ambient wind (referred to as the No Shear case). As seen in Fig. 7, the

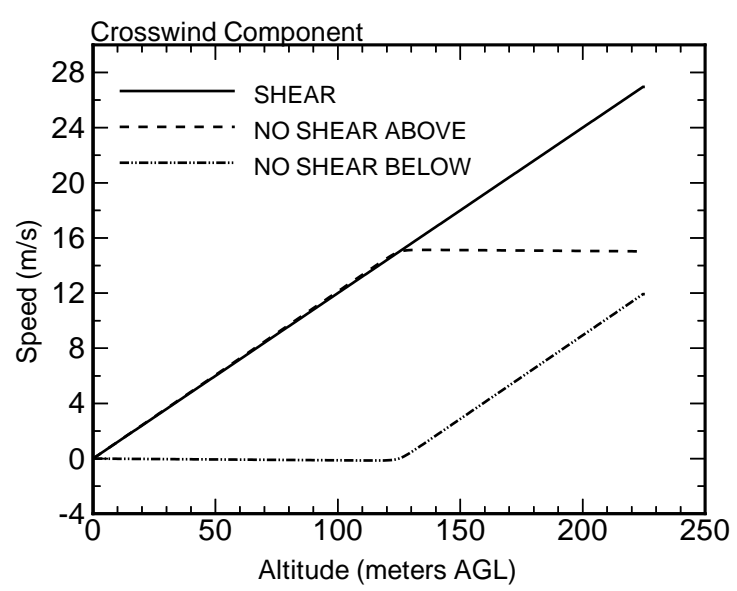

Figure 7. Crosswind profiles used as input for crosswind shear experiments.

constant linear shear case (Shear) has a crosswind profile that increases from no wind at the ground to $30 \mathrm{~m} / \mathrm{s}$ at $250 \mathrm{~m}$. This gives a constant magnitude of shear of $0.12 \mathrm{~s}^{-1}$. Also examined are two transitional cases, which assume an environment that transitions between constant shear and no shear. The wind profile for the "No Shear Above" case is identical to the linear-shear case, except that it transitions to a constant crosswind of $15 \mathrm{~m} / \mathrm{s}$ above an altitude of $125 \mathrm{~m}$.. On the other hand, the "No Shear Below" case has no crosswind below $125 \mathrm{~m}$, but an increasing crosswind above this height with the same magnitude of shear as in the linear-shear case. In all cases the initial wake vortex is generated at an altitude of $175 \mathrm{~m}$, which is $50 \mathrm{~m}$ above the transition altitude.

Time height profiles of the vortex trajectories are show for the port (upstream) vortex (Fig. 8) and the starboard (downstream) vortex (Fig. 9). The altitude vs lateral position trajectories are shown in Fig. 10. Note that the transition from shear to no shear and vice versa has a pronounced effect on the descent rate of the vortex trajectories. On the other hand, the descent rate for the vortex embedded in the linear shear was nearly identical to that of the vortex pair embedded in the no shear (no crosswind) environment. The trajectories of the vortex pair in the linear shear environment (Fig. 10) are convex in shape due to the decreasing wind speeds at lower altitudes. However, as for reasons described in the previous set of experiments, the vortex pair at lower levels translates faster than the mean winds for which they are embedded. Descent rates for the linear shear case were identical for both upstream and downstream vortex. Tilting of the vortex pair was not apparent. 
Comparison of Figs. 7 and 8 show that the upstream vortex bounced highest when the shear is aloft, while downstream vortex bounces highest when the shear is near the ground. As indicated in Table 3, the bounce of the vortex is related to the vertical change in ambient shear (i.e. $\partial^{2} U / \partial z^{2}$ ) rather than the environmental shear alone. In terms of the vorticity due to the ambient crosswind (crosswind vorticity), $\partial U / \partial z$, we see that the vortex with a sign of vorticity opposite to the sign of the vertical change in ambient vorticity, bounces highest. From these experiments, we see that vertical changes in the along-track component of ambient vorticity can preferentially reduce the descent rate of vortices, and may lead to vortex tilting and vortex rising. Linear shear (constant ambient vorticity) has no impact on the vortex descent rates.

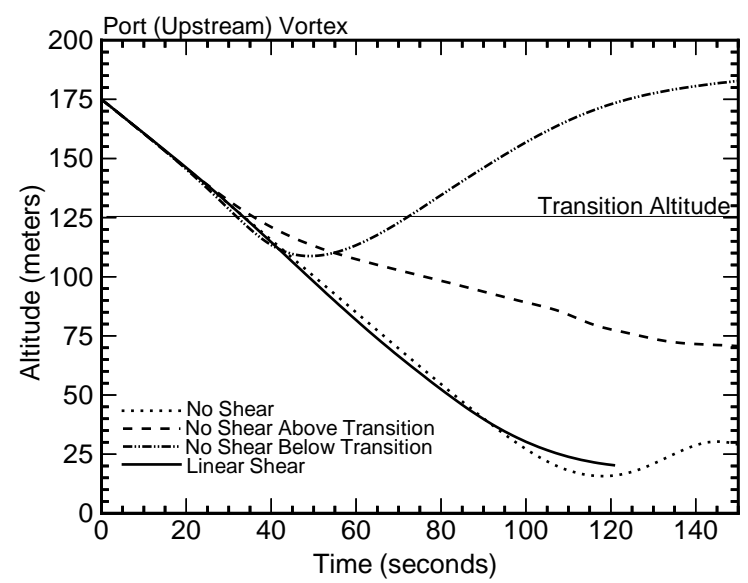

Figure 8. Time-height trajectories for upstream vortex in crosswind shear experiments.

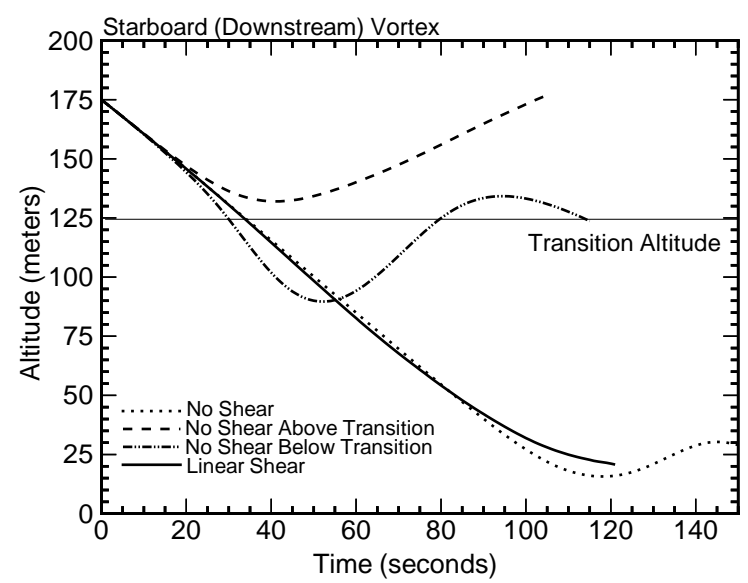

Figure 9. Same as Fig. 8, but for downstream vortex.

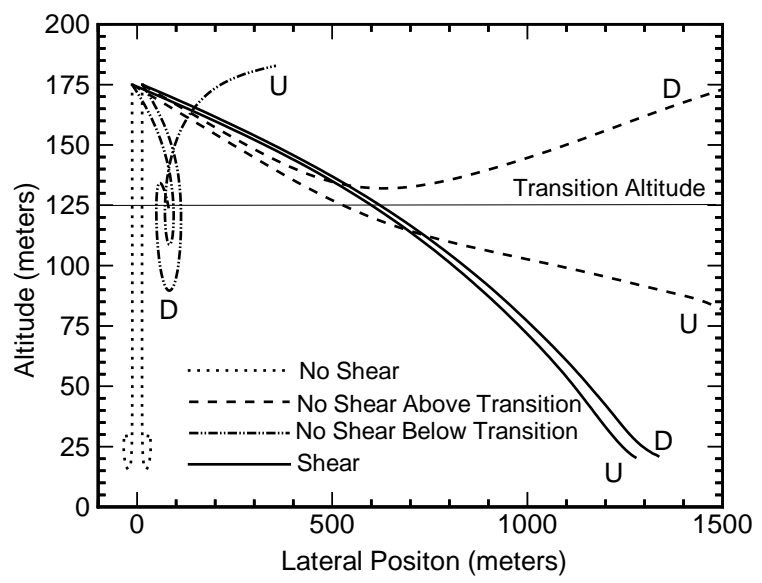

Figure 10. Same as Fig. 4, but for crosswind shear experiments.

Table 3. Sign of crosswind vorticity vs vortex with highest bounce for each experiment

\begin{tabular}{lccc}
\hline Case & $\begin{array}{c}\text { Vorticity } \\
\omega\end{array}$ & $\partial \omega / \partial z$ & $\begin{array}{c}\text { Vortex with } \\
\text { Highest } \\
\text { Bounce }\end{array}$ \\
\hline No shear & 0 & 0 & same \\
Linear shear & + & 0 & same \\
No shear above & + & - & downstream \\
No shear below & + & + & upstream \\
\hline
\end{tabular}

\section{IDF run 9 Case.}

To give supporting confirmation to the above shear study, we have chosen a former TASS validation case $^{4}$ which compares simulated with measured trajectories. Input values for the simulation were based on known aircraft parameters and observed vertical distributions for ambient temperature and wind. Field measurements of wake vortex generated from a $B-757$ 200 (sponsored by the FAA) were taken at the Idaho Falls Field Center. The test aircraft was on a level flight at $70 \mathrm{~m}$ above ground level (AGL) in an early morning fly-by. The observed environmental profiles for potential temperature and crosswind are shown in Fig. 11. Note from the sounding that there is a pronounced zone of crosswind shear with a peak magnitude of $0.1 \mathrm{~s}^{-1}$ located between 25 and $50 \mathrm{~m}$ AGL. The magnitude of the 
crosswind change across this zone is slightly under $3 \mathrm{~m} / \mathrm{s}$. Measured data for this case was obtained from an instrumented tower, ${ }^{24}$ Laser Doppler Velocimeter (LDV) and a Monostatic Acoustic Vortex Sensing System (MAVSS). ${ }^{25,26}$

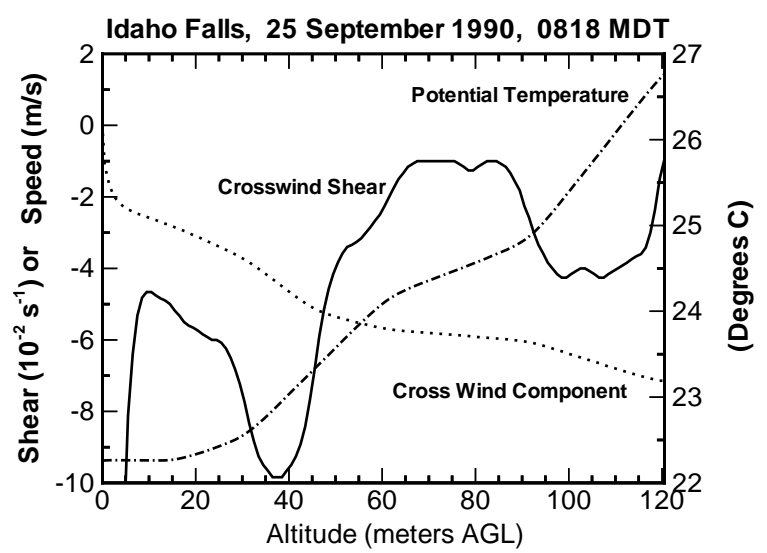

Figure 11. Input sounding for Idaho Falls Run 9. Curves are for potential temperature (dash dot), crosswind (dotted) and crosswind shear (solid).

Both the TASS model prediction and measured data show that the trajectory of the downstream (port) vortex is deflected as it encounters the zone of crosswind shear between 25-50 m AGL (Fig. 12). The crosswind vorticity in this shear region has opposite sign to that of the downstream vortex, while the change in vorticity with

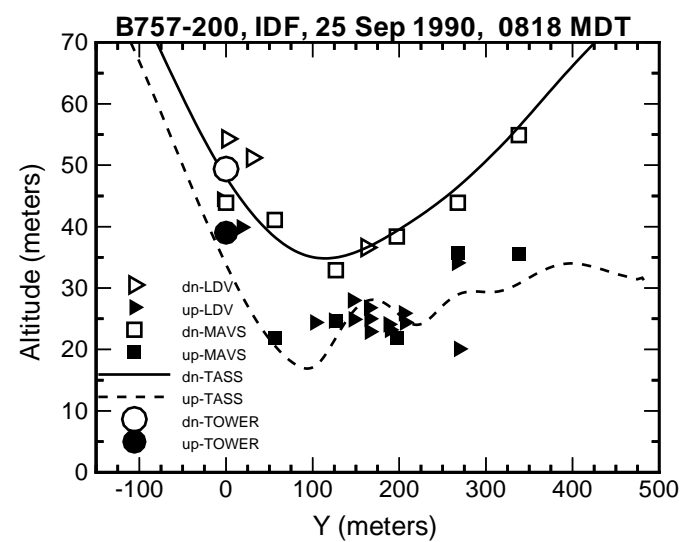

Figure 12. Comparison of TASS predicted trajectories with filed data for Idaho Falls, B-757, Run 9 case. Field measurements are delineated by open (downstream vortex) and filled (upstream vortex) symbols. Model predictions are delineated by solid (downstream) and dashed (upstream) lines. height at the top of this layer is of the same sign as the vorticity in the downstream vortex. This interaction of the downstream vortex with the crosswind vorticity shear of the same sign eventually causes it to rise upward with increasing lateral separation from the upstream (starboard) vortex.

\section{Sensitivity to Initial Turbulence}

In a large eddy simulation of wake vortices, turbulence can be divided into several categories which include: 1) subgrid-scale turbulence, 2) resolvable-scale turbulence generated by the interaction of the vortex pair, 3) resolvable-scale turbulence generated by the interaction of the wake vortex with the ground and its environment, and 4) preexisting resolvable-scale turbulence. The simulations presented in the previous sections have ignored the existence of the latter category. In order to evaluate its sensitivity, we have developed a procedure for initiating background turbulence into our model. Turbulence initialization is accomplished through the use of a random streamfunction field in conjunction with a low-pass filter. The use of the streamfunction ensures incompressibility of the turbulent velocity field while the low-pass filter prevents the initial turbulent energy from being concentrated at the smallest scales. The three-dimensional streamfunction and velocity vectors are defined as:

$$
\begin{gathered}
\vec{\psi}=\left(\psi_{x}, \psi_{y}, \psi_{z}\right) \\
\overrightarrow{\boldsymbol{V}}=\nabla \times \vec{\psi} \\
\nabla \cdot \vec{V}=\nabla \cdot \nabla \times \vec{\psi} \equiv 0
\end{gathered}
$$

In two dimensions, this reduces to:

$$
\begin{gathered}
\Psi=\left(0, \psi_{y}, 0\right) \\
u^{\prime}=-\frac{\partial \Psi_{y}}{\partial z} \\
w^{\prime}=\frac{\partial \psi_{y}}{\partial x}
\end{gathered}
$$

where $u^{\prime}$ and $w^{\prime}$ are the added crosswind and vertical velocity fluctuations due to grid-scale turbulence.

At each grid point, $\psi_{\mathrm{y}}$ is assigned a random number with a prescribed standard deviation. It is desirable to avoid having the largest velocity variations concentrated at the smallest scales, since that energy is quickly dampened by subgrid turbulence. Thus, a nine point filter is used to smooth the streamfunction before it is differentiated. An approximate relationship between the standard deviation of the streamfunction and the turbulence kinetic energy is used to prescribe the latter as 
a function of height. After an initial development period, two-dimensional vortices are initialized by adding the usual initial vortex field to the background turbulent velocity field.

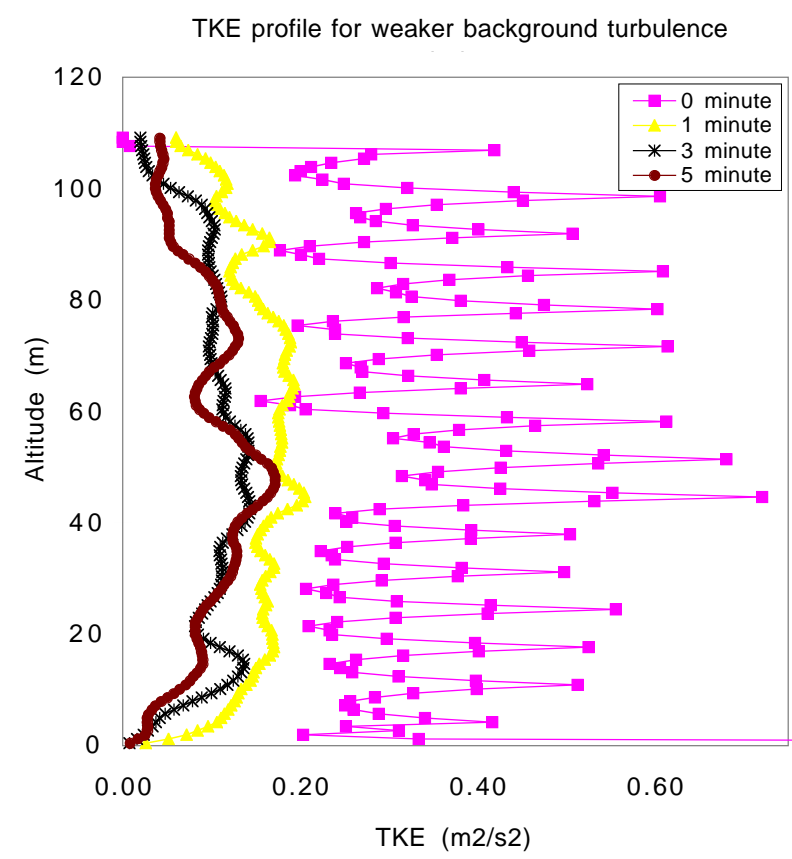

Figure 13. Turbulence kinetic energy profiles for background turbulence at several model simulation times.

In an initial test, we avoid the complicating effect of background wind shear and stratification by assuming that the atmosphere is neutrally stable and there is no background mean wind. We have chosen two strengths of turbulence for this case. The first case, labeled as "weaker turbulence," employs turbulence which is typical of convective conditions in the atmosphere (Stull, ${ }^{16}$ pp. 359 and 371). The second case uses an order magnitude stronger turbulence than the first. The temporal variation of the turbulence kinetic energy profiles for the weaker case is shown in Fig. 13. In each case, the profile becomes smooth and nearly steady in time after about three minutes. It was at three minutes that vortices were initialized in the turbulent runs.

A vortex pair without background turbulence initialization (baseline run) descends initially due to the mutual interaction of their velocity fields with a constant initial separation. When they approach the ground, secondary vortices are induced due to the production of countersign vorticity at no-slip boundaries and force the primary vortices to rise up. Interactions with secondary vortices result in a spiral path of the primary vortices. Four different random number generations were used in separate runs for both the weaker and stronger turbulence cases. Because of the symmetric nature of the flow in these cases with no background wind, this results in eight vortex trajectories for each turbulence strength from which we calculated averages and standard deviations. Time histories of lateral position, and altitude for the weaker turbulence case are shown in Fig. 14. A single curve is shown for the case without resolved turbulence, while three curves are shown for the turbulent case representing the mean, the mean plus one standard deviation, and the mean minus one standard deviation. We see from the figure that the resolved background turbulence has very little effect on the vortex transport in an ensemble sense. The main effect is a smaller amplitude of the oscillations caused by the secondary vortices. Although not shown, the effect on average circulation from including the initial background turbulence is even smaller.

Fig. 15 shows the same plots for ten times stronger turbulence. Here the results are similar but with two exceptions. The first is a larger spread for the positions for the turbulent runs. This indicates than any individual vortex history could be significantly different with very strong turbulence, but the average behavior is nearly the same. The second exception is a tendency for less lateral transport with very strong resolved turbulence after two minutes.

These results suggest that two-dimensional background turbulence does not play a significant role in vortex decay. Some well-known decay mechanisms, such as Crow instability and vortex bursting, are highly three-dimensional and cannot occur in a two-dimensional flow. Regarding wake transport, the turbulent eddies appear to cause stochastic variations in the vortex motions which become significant only for late times and very strong turbulence. In addition, the effect of the ground-induced secondary vortices appears to be somewhat weakened.

The turbulence field initialization was applied to several of the validation cases from the Idaho Falls field study. Values for the initial turbulence were estimated based on the turbulence velocity scale for the convective boundary layer ${ }^{27}$ as derived from the observed sounding for each case. Cases were also run using 10 times the estimated value for turbulence. In these cases, no appreciable improvement was gained by including resolved two-dimensional turbulence. The strength of 

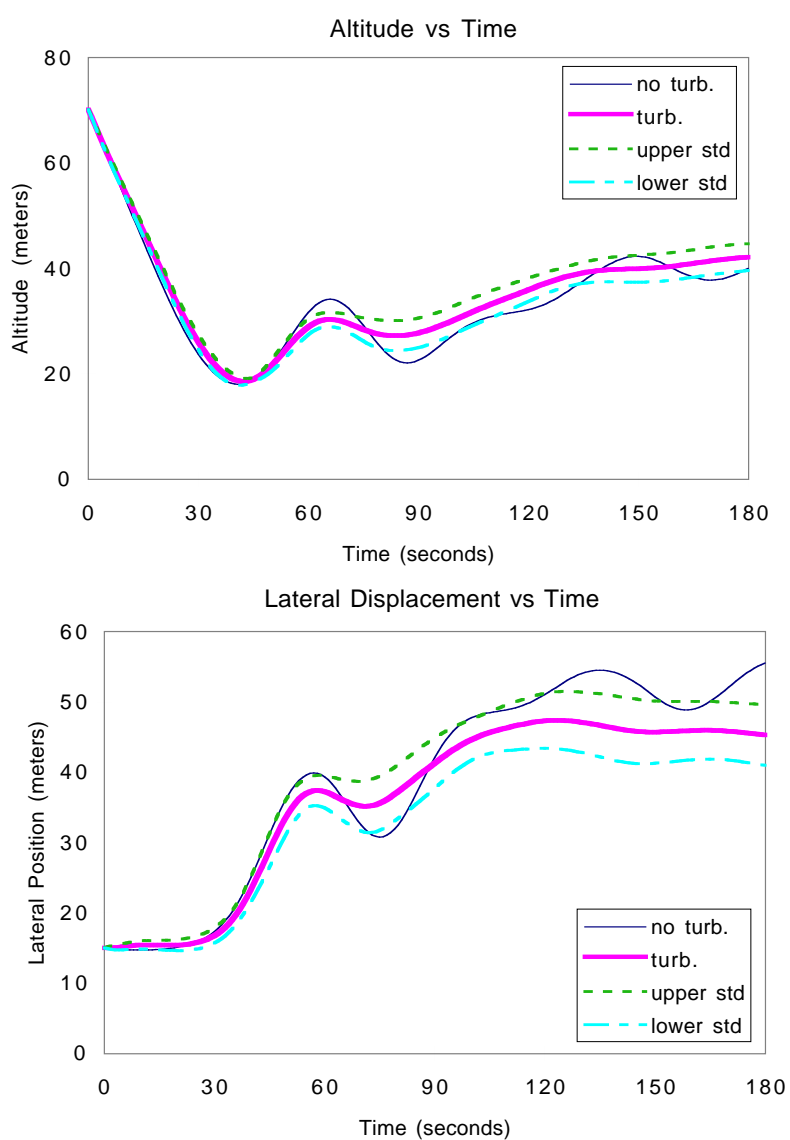

Figure 14. A comparison of wake vortex behavior with and without weak resolved background turbulence. The mean (turb.), the mean plus the standard deviation (upper std.), and the mean minus the standard deviation (lower std.) are shown. (a) Altitude vs time; (b) Lateral position vs time.

the turbulence had virtually no effect on the 10-meter average circulation history.

In summary of the turbulence initiation experiments, moderately strong two-dimensional background resolved turbulence has, in general, a minor effect on wake vortex transport. Very strong turbulence, however, can result in significant chaotic motion of the individual vortices. Background resolved turbulence has insignificant effects on the 10-meter average circulation. However, we expect to see a much more pronounced effect when it is included in 3-D simulations of the wake vortices, since turbulence is more realistically treated and 3-dimensional vortex instabilities would be permitted.

\section{Summary}

Two-dimensional TASS simulations are shown to be useful for investigating the effects of stratification, wind shear, and turbulence on wake vortex transport. Simulations presented in this paper confirm that conventional nondimensionalizations work well for stratified environments in the absence of shear. The usefulness of their application to more complex environments is yet to be evaluated.
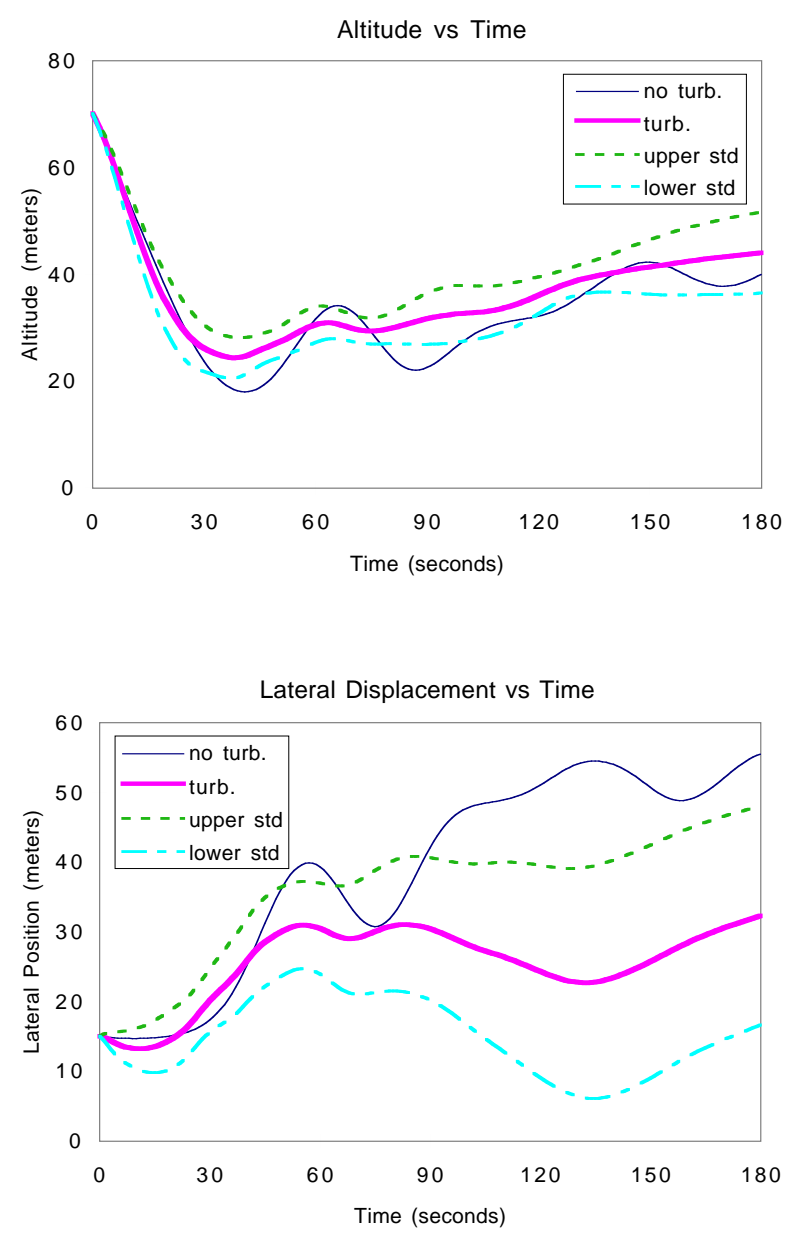

Figure 15. Same as Fig. 14, but for strong turbulence.

Our results show that wake vortex trajectories are very sensitive to the crossflow component of the wind. Changes in crosswind with altitude can affect the wake vortex trajectories in several ways. As a vortex descends through a sheared crossflow its lateral speed is affected, but may be different from the speed of the crossflow at a lower level. This is partly due to the downward transport of crossflow momentum from its generation point. Nonlinear shear of crossflow affects the vortex descent rates and may result in vortex tilting or 
rising. The member of the vortex pair with vorticity of same sign as the vertical change in the ambient alongtrack vorticity, may be deflected upwards. These effects appear to be more sensitive than the affects of stable stratification. Zones with sharp vertical changes in the crosswind, like that associated with fronts or inversions, may be quite effective in altering the vortex trajectories. Detection of crossflow distributions may be critical to AVOSS and other similar prediction systems. This will require special sensor to monitor the winds aloft in the terminal area.

Our results also suggest that stable stratification must be quite strong in order to have the same affect as crosswind shear. Aircraft such as the large wide-body jetliners may be more susceptible to the stratification effect than small and medium range jet aircraft.

A procedure for initiating wake vortex simulations with grid-scale turbulence was described. For the cases examined, the addition of background resolved-scale turbulence had only minor effects on twodimensional vortex transport. However, in threedimensional simulations with unstable atmospheres, we expect significant sensitivity to background, resolvedscale turbulence.

\section{$\underline{\text { Acknowledgements }}$}

This research was sponsored by NASA's Terminal Area Productivity Program. Contributions by authors from North Carolina State University were sponsored under NASA Cooperative Agreement NCC-1-188. Numerical simulations were carried out on NASA Langley's Cray YMP and NASA Ames' Cray 90 supercomputers.

\section{References}

1. Hinton, D.A., "Aircraft Vortex Spacing System (AVOSS) Conceptual Design," NASA Tech Memo No. 110184, August 1995.

2. Hinton, D.A., "An Aircraft Vortex Spacing System (AVOSS) for Dynamical Wake Vortex Spacing Criteria, "AGARD 78th Fluid Dynamics Panel Meeting \& Symposium, Trondheim, Norway, Paper No. 23, May 1996.
3. Proctor, F.H., "The Terminal Area Simulation System. Volume I: Theoretical Formulation," NASA Contractor Rep. 4046, DOT/FAA/PM-86/50, I, April 1987. [Available from NTIS]

4. Proctor, F. H., "Numerical Simulation of Wake Vortices Measured During the Idaho Falls and Memphis Field Programs," 14th AIAA Applied Aerodynamics Conference, Proceedings, Part-II, New Orleans, LA, AIAA Paper No. 96-2496, June 1996, pp. 943-960.

5. Schowalter, D.G., DeCroix, D.S., Switzer, G.F., Lin, Y.-L., and Arya, S.P., "Toward Three-Dimensional Modeling of a Wake Vortex Pair in the Turbulent Boundary Layer," AIAA 97-0058, January 1997.

6. Turner, J.S., "A Comparison Between Buoyant Vortex Rings and Vortex Pairs," J. Fluid Mech. Vol 7, 1960, pp. 451-464.

7. Scorer, R.S. and Davenport, R.S., "Contrails and Aircraft Downwash," J. Fluid Mech., Vol 43, 1970, pp. 451-464.

8. Tombach, I., "Observations of Atmospheric Effects on Vortex Wake Behavior," J. Aircraft, Vol 10, 1973, pp. 641-647.

9. Hill, F.M.., "A Numerical Study of the Descent of a Vortex Pair in a Stably Stratified Atmosphere," J. Fluid Mech., Vol 71, 1975, pp. 1-13.

10. Bilanin, A.J., Teske, M.E., and Hirsch, J.E., "The Role of Atmospheric Shear, Turbulence and A Ground Plane on the Dissipation of Aircraft Vortex Wakes," AIAA Journal, Vol 16, 1978, pp. 956-961.

11. Hecht, A.E., Bilanin, A.J., and Hirsch, J.E., "Turbulent Trailing Vortices in Stratified Fluids," AIAA Journal, Vol 19, 1981, pp. 691-698.

12. Sarpkaya, T., "Trailing Vortices in Homogeneous and Density-Stratified Media," J. Fluid Mech., Vol 136, 1983, pp. 85-109.

13. Greene, G.C., "An Approximate Model of Vortex Decay in the Atmosphere," J. Aircraft, Vol 23, 1986, pp. 566-573. 
14. Robins, R.,E. and Delisi, D.P., "Numerical Study of Vertical Shear and Stratification Effects on the Evolution of a Vortex Pair," AIAA Journal, Vol 28, 1990, pp. 661-669.

15. Spalart, P.R., "On the Motion of Aircraft Wakes in a Stably Stratified Fluid," to appear in J. Fluid Mech., 1996.

16. Stull, R.B., An Introduction to Boundary Layer Meteorology, Kluwer Academic Publishers, 1988.

17. Brashears, M.R., Logan, N.A., and Hallock, J.N., "Effect of Wind Shear and Ground Plane on Aircraft Wake Vortices," J. Aircraft, Vol 12, 1975, pp. 830833.

18. Robins, R.E., and D.P. Delisi, "Potential Hazard of Aircraft Wake Vortices in Ground Effect with

Crosswind," J. Aircraft, Vol 30, 1993, pp. 201-206.

19. Zheng, Z.C., and Ash, R.L., "Study of Aircraft Wake Vortex Behavior Near the Ground," AIAA Journal, Vol 34, pp. 580-589.

20. Delisi, D.P., Robins, R.E., and D. Lucas, “ Initial Laboratory Observations of the Evolution of a Vortex Pair in a Stratified Shear Flow," Phys. Fluids, Vol 3, 1991, pp. 2489-2491.

21. Schilling, V.K., "Motion and Decay of Trailing Vortices within the Atmospheric Surface Layer," Beitr. Phys. Atmosph., Vol 65, No. 2, 1992, pp. 157-169.

22. Switzer, G.F., "Validation Tests of TASS for Application to 3-D Vortex Simulations," NASA Contractor Report 4756, 1996.

23. Matthews, M.P., Dasey, T.J., Perras, G.H., and Campbell, S.D., "Planetary Boundary Layer Measurements for the Understanding of Aircraft Wake Vortex Behavior," 7th Conf. on Aviation Weather Systems, Long Beach, CA, Amer. Meteor. Soc., February 1997.

24. Garodz, L.J., and Clawson, K.L., "Vortex Wake Characteristics of B757-200 and B767-200 Aircraft Using the Tower Fly-By Technique, Volumes 1 and 2," NOAA Tech. Memo. ERL ARL-199, Jan. 1993.

[Available from NTIS]
25. Systems Research Corporation, "Atmospheric Description for Idaho Falls B-757 Run \#9 on September 25, 1990," Volpe National Transportation Systems, Cambridge, MA, Jan. 1994.

26. Rudis, R.P., Burnham, D.C. and Janota, P., "Wake Vortex Decay Near the Ground Under Conditions of Strong Stratification and Wind Shear," 78th Fluid Dynamics Panel Meeting \& Symposium, Trondheim, Norway, 20-23, May 1996.

27. Arya, S.P., "The Atmospheric Boundary Layer and its Parameterization," Wind Climate in Cities, J. E. Cermak, A. G. Davenport, E. J. Plate, and D. X. Viegas, Eds., Kluwer Academic Publishing, 1995, pp 41-66. 